\title{
Estimation of left ventricular filling pressure post cardiac surgery: echocardiography VS. invasive measurement of left atrial pressure (ECHOPOG study)
}

\author{
T.Wipf', G.Ajob', P. G. Guinot ${ }^{2}$, O. Collange', W. Oulehri', P. M. Mertes' \\ I Hôpitaux Universitaire de Strasbourg - Strasbourg (France), 2 CHU Dijon - Dijon (France)
}

\section{Background and Goal of Study:}

Measurements of echo-Doppler parameters such as the transmitral flow (E/A) and early diastolic velocity of mitral annulus (Ea) are used to assess left ventricular filling pressure (LVFP) and thus guide fluid therapy. These parameters, although of daily use in the ICU, have never been properly evaluated on a homogeneous population of post-cardiac surgery patients. We designed this study to test the reliability of non-invasive LVFP estimation in the ICU after cardiopulmonary bypass (CPB).

\section{Materials and Methods:}

We conducted a single-center, prospective observational study at a University Hospital. All adult patients admitted in the ICU after cardiac surgery with CPB were considered eligible for participation. Patients who had undergone mitral surgery or with mitral pathology were excluded. Simultaneously with Trans Thoracic Echography (TTE), left atrial pressure (LAP) measurement was performed through a surgically placed catheter. LAP was considered as the reference measurement for LVFP. Echo-Doppler measurements $\left(E / A, T D E, E / E a, E / V_{P}\right)$ were performed offline without knowledge of the hemodynamic data. The primary study endpoint was the correlation between LAP and E/Ea. Secondary study endpoints were the correlation between LAP and other echo-Doppler parameters ( $E$ wave, E/A, TDE, E/VP) and their diagnostic performances, isolated or combined through the 2016 algorithm of the American Society of Echocardiography (ASE), to predict a LAP> $18 \mathrm{mmHg}$.
Results and Discussion:

62 patients and 88 trans-thoracic echography (TTE) were analyzed.

We observed no significant correlation between $\mathrm{E} / \mathrm{Ea}$ and LAP (Pearson's coefficient at 0,19; $=0,08$ ).

Subgroup analysis according to ventilatory mode, cardiac rhythm or LVEF did not change this result. Other parameters were significantly but weakly correlated with LAP: $E$ wave $(r=0.44 ; p<0.00 I)$, E/A $(r=0.36 ; p=0.00 I)$, DTE $(r=-0.26 ; p=0.016)$.

The E/Ea ratio could predict a LAP> $18 \mathrm{mmHg}$ with a $46 \%$ sensibility and a $83 \%$ specificity, with a best cut-off point value at $13 \mathrm{~cm} / \mathrm{s}$.

The application of the 2016 ASE algorithm did not improve this performance with a $50 \%$ sensibility and a $75 \%$ specificity to predict a LAP> $18 \mathrm{mmHg}$.

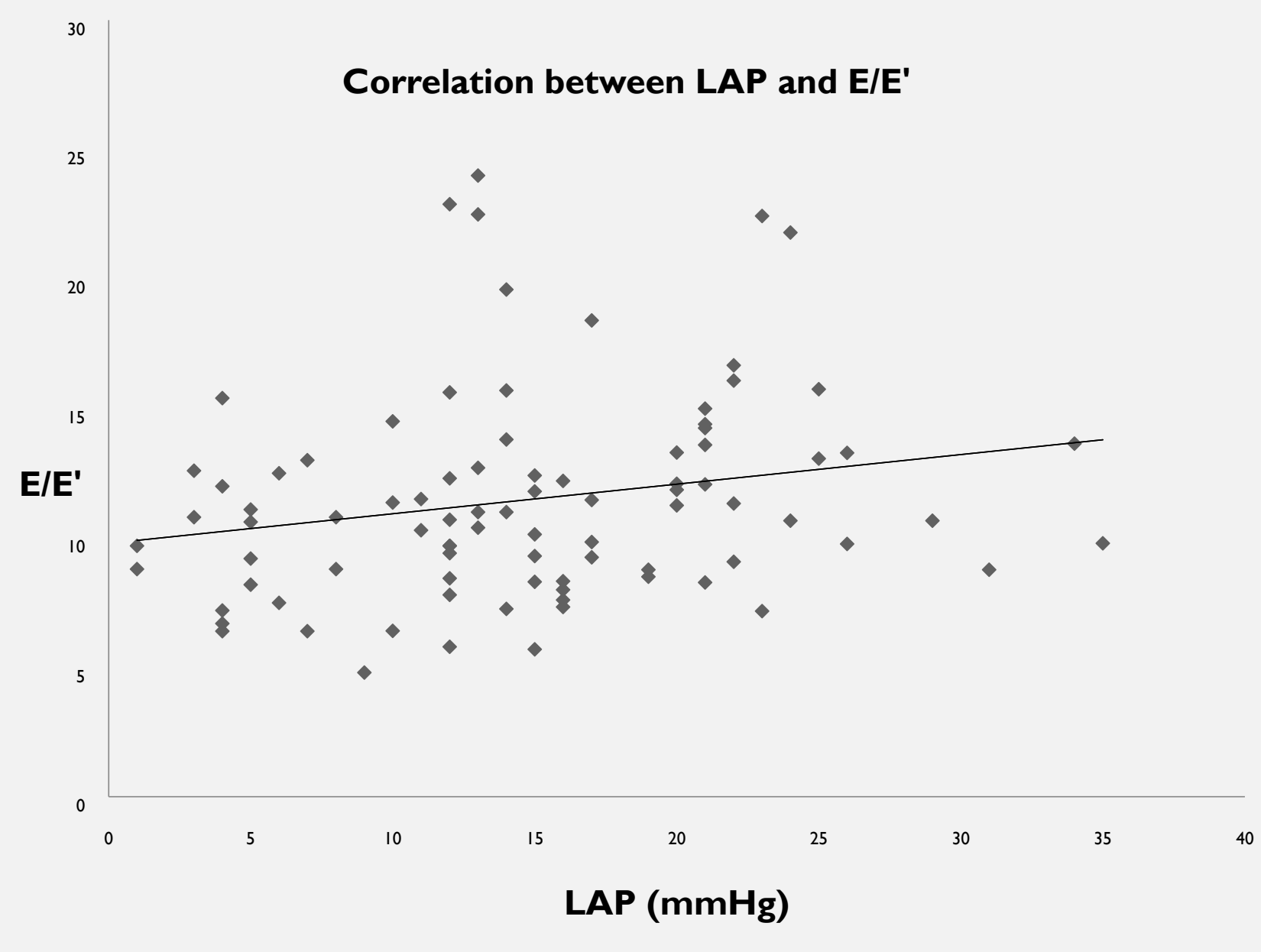

\section{Conclusion(s):}

Our results suggest that post-cardiac surgery TTE cannot be reliably used for the LVEP

assessment in the ICU, regardless of whether the echoDoppler parameters are isolated or combined using the 2016 ASE algorithm. 